\title{
Diagnosis of Faraday Rotation with Video Vector Magnetograph at Huairou
}

\author{
Jiangtao Su and Hongqi Zhang \\ National Astronomical Observatories, Chinese Academy of Sciences, \\ Beijing 100012, China
}

Over the wavelength interval of $150 \mathrm{~m} \AA$ redward of line center of the FeI $\lambda 5324.19 \AA$ to $150 \mathrm{~m} \AA$ to the blue, in steps of $10 \mathrm{~m} \AA$, observations of a simple sunspot were obtained with the Video Vector Magnetograph at Huairou Solar Observing Station (HSOS) of National Astronomical Observatories. In this paper, we present results of the inversion of stokes polarization profiles of this sunspot to recover the vector magnetic field parameters of the spectral line forming region using the FeI $\lambda 5324.19 \AA$ using a nonlinear least-squares fitting. At the same time, curves of the observed variation of azimuth with wavelength were compared with model calculations for the azimuth at each wavelength as derived the inverse Zeeman effect modified by Faraday rotation. Hagyard, et al. (2000, Solar Phys. 191, 309) find it is difficult to separate two effects of Faraday rotation and $\pi-\sigma$ rotation. However, $\pi-\sigma$ rotation relates to the Landé factor of a spectral line. For the spectral line FeI $5324.19 \AA$, its Landé factor $g=1.5$. In the solar magnetic field atmosphere, its Zeeman split is less than that of line FeI $5250.22 \AA$ which has larger Landé factor $\mathrm{g}=3$. The correlations in Figure 1 show that the azimuth rotation increases as the field strength increases and inclination angle decreases. The results show that rotation of the azimuth is less significant in observation taken near the center of the FeI $\lambda 5324.19 \AA$ than that of FeI $\lambda 5252.22 \AA$ line.
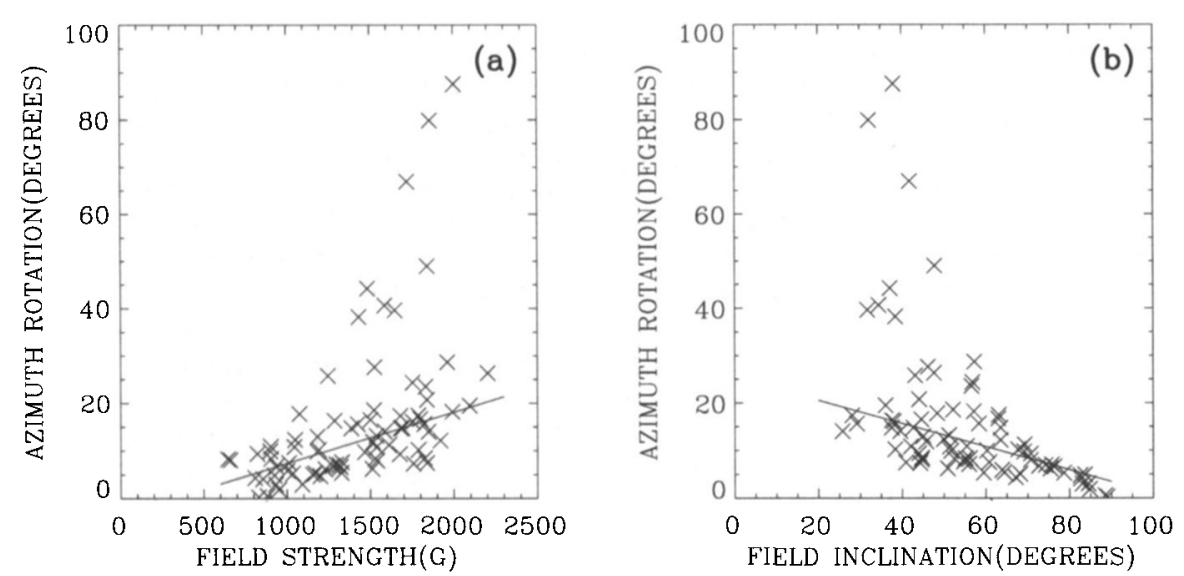

Figure 1. Correlation plots of field strength and field inclination 